\title{
Si près de chez vous : produire du fait divers à la chaîne
}

Si près de chez vous: Assembly Line Production of Crime News Items

\section{Amélie Chabrier}

\section{(2) OpenEdition}

\section{Journals}

\section{Édition électronique}

URL : http://journals.openedition.org/recherchestravaux/977

DOI : 10.4000/recherchestravaux.977

ISSN : 1969-6434

Éditeur

UGA Éditions/Université Grenoble Alpes

\section{Édition imprimée}

ISBN : 978-2-37747-056-3

ISSN : 0151-1874

\section{Référence électronique}

Amélie Chabrier, "Si près de chez vous : produire du fait divers à la chaîne », Recherches \& Travaux [En ligne], 92 | 2018, mis en ligne le 01 juin 2018, consulté le 23 septembre 2020. URL : http://

journals.openedition.org/recherchestravaux/977 ; DOI : https://doi.org/10.4000/recherchestravaux. 977

Ce document a été généré automatiquement le 23 septembre 2020.

(c) Recherches \& Travaux 


\section{Si près de chez vous : produire du fait divers à la chaîne}

Si près de chez vous: Assembly Line Production of Crime News Items

\section{Amélie Chabrier}

JEANNE : «Tu peux m'expliquer ça ? Non?

T'as décidé de me ridiculiser?» Dans les dernières parutions du journal, Jeanne a eu la

désagréable surprise d'y voir étalée sa relation d'amour passée avec Sébastien. L'auteur y puise son inspiration. SÉBASTIEN : « Ça va, y’a rien de grave. C'est vrai, je me suis un peu servi de notre histoire, mais y'a vraiment pas de quoi fouetter un chat !

JEANNE : Tu plaisantes Sébastien ! Mais... mais c'est le succès qui te monte à la tête !

SÉBASTIEN : Mais c'est une fiction Jeanne! Personne ne fera jamais le rapprochement entre toi et cette histoire!

" L'Homme derrière les mots ", Si près de chez vous, Saison 1, 2012.

1 Le discours médiatique est un vaste ensemble qui regroupe, selon Dominique Maingueneau, plusieurs types: le discours radiophonique, le discours de presse écrite, le discours télévisuel ${ }^{1}$. Différents genres composent ces types: étudier un genre médiatique revient à identifier des dispositifs de communication liés à des conditions socio-historiques, autour d'un média particulier. Le fait divers comme genre routinier est lié à l'émergence d'une presse bon marché, grand public, qui apparaît en France dans les années $1865^{2}$. Il trouve ses prémices dans d'autres formes médiatiques archaïques, la rumeur et le canard, et connait par la suite des adaptations pour d'autres médias, comme la radio, la télévision ou le web33. 
2 Pour circonscrire un genre, on s'intéresse donc aux conditions socio-historiques qui l'ont favorisé, à son histoire, mais aussi aux genres limitrophes hébergés par un même support: la présence de fictions au sein du journal au XIX ${ }^{\mathrm{e}}$ siècle entraîne une hybridation et une circulation des formes, d'où le terme de littérarisation employé pour désigner des procédés venus de la littérature et réemployés dans un article ${ }^{4}$. Cet échange n'est d'ailleurs pas à sens unique: la forme de l'enquête, la personne du journaliste investigateur mise en scène dans le reportage, entraînent la création d'un nouveau genre: le roman policier ${ }^{5}$. Ce réseau dense de formes hybrides, de textes factuels, mi-factuels, mi-fictionnels, fictionnels, constitue ce qu'Alain Vaillant désigne par le terme de littérature médiatique ${ }^{6}$, dans une définition résolument ouverte, communicationnelle, de la littérature ${ }^{7}$ : celle-ci se définit comme «l'ensemble des textes qui ne sont pas d'usage professionnel ni pratique et qui sont mis en libre circulation dans l'espace public ", grâce à la communication médiatique moderne telle qu'elle se met en place à partir du XIX ${ }^{\mathrm{e}}$ siècle.

3 On pourrait alléguer que les faits divers ne sont lus, ne sont lisibles, que dans leur contexte, ce qui restreint leur public. Pourtant rien n'est moins sûr : en témoignent les recueils de faits divers qui fleurissent chaque année en librairie sur les mystères de telle année, ou de telle région. Contrairement à des articles politiques par exemple, comme Roland Barthes l'avait bien remarqué, l'immanence du fait divers est la garantie d'un public ouvert, d'un usage non professionnel, bref d'une inscription au cœur de la littérature médiatique ${ }^{8}$. Les affaires criminelles, - mais aussi les petits faits divers banals ou incongrus - passionnent dans leur actualité mais également après, parce qu'ils gardent justement la caution du réel : la télévision l'a bien compris, qui propose des magazines, des documentaires fictionnels (docu-fictions), des téléfilms reconstituant des faits divers passés, historiques ou ultra récents (docu-dramas), quitte à s'en voir interdire la diffusion'. Parce qu'il est avant tout mise en récit, le fait divers ne cesse de poser la question de la frontière entre fiction et réel, les différents genres médiatiques qui s'en emparent ne faisant que moduler ce rapport. Le fait divers à la télévision, outre celui du journal télévisé, se présente donc sous des formes multiples, elles-mêmes influencées par des genres audiovisuels limitrophes sur la grille des programmes. Tous les spécialistes du média notent ainsi les modifications profondes que la téléréalité a apportées aux genres télévisuels à partir des années $2000^{10}$, la conséquence étant, selon Annabelle Klein et Axel Tixhon, que :

[...] l'un des traits marquants de l'écriture audio-visuelle actuelle est précisément le déplacement des frontières entre le réel et la fiction. On voit des créations fictionnelles utilisant des traits caractéristiques du documentaire et, inversement, des documentaires largement mis en scène. Les différents genres se télescopent ${ }^{11}$.

Ces réflexions préalables serviront à l'étude d'un genre télévisuel récent et a priori éphémère: la scripted reality, aussi désignée sous l'expression francisée de «réalité scénarisée » ou « fiction du réel ». Comme très souvent à la télé, il s'agit d'un concept importé d'un autre pays par des producteurs, en l'occurrence Julien Courbet qui s'inspire en 2011 de la télé allemande pour lancer une première émission sur France 2. L'oxymore exhibe d'emblée sa nature mixte, sa tension entre réel et fiction. Suite à ce premier essai, l'année 2012 voit fleurir sur plusieurs chaînes (TF1, M6, NRJ 12, France Télévisions) ce genre, qui se décline en plusieurs programmes : Au nom de la vérité, Dans la tête du tueur, Derniers Recours, Hollywood Girls, Le jour où tout a basculé, Le jour où tout a basculé... à l'audience, Mon histoire vraie, Soupçons, Une histoire, une urgence, Par amour, Petits Secrets entre voisins, Petits Secrets en famille, Face au doute ${ }^{12}$. Nous choisirons Si près de chez 
vous pour cette étude du genre : diffusée sur France 3 entre 2012 et 2014, produite par 909 Productions et faisant intervenir de nombreux auteurs et scénaristes français, $\mathrm{Si}$ près de chez vous est une série qui met en scène de petits faits divers criminels réels résolus. Agressions, chantage, héritage, adultère, jalousie, vol, homicide, assassinat, chaque intrigue se situe dans le quotidien le plus banal, dans l'infra-ordinaire ${ }^{13}$. Elle compte deux cent trente-cinq épisodes, et nous puiserons nos exemples dans les deux saisons. Le concept est le suivant : "mettre en scène des histoires vraies ou des faits divers et les faire jouer par des comédiens ${ }^{14} »$. Ce qui brouille les pistes de façon nouvelle - mais qui s'inscrit dans une pratique d'hybridation générique généralisée à la télévision de ces dernières années -, est que la réécriture, le script, utilise les codes, les styles de plusieurs médias consacrés au fait divers:

Le délitement des frontières et des genres, amèn[e] le téléspectateur d'aujourd'hui à naviguer constamment entre les codes et à se positionner face aux productions audio-visuelles brouillant sciemment les pistes de la fiction et du réel. [...] [N]otre paysage médiatique renvoie aujourd'hui à un incessant travail de décodage et de positionnement face à ces nouvelles formes de productions audio-visuelles ${ }^{15}$.

Cet « à la manière de » n'est pas sans poser de questions : les instances de l'audiovisuel ont longuement débattu en 2013 sur cet objet nouveau, oscillant entre programme de flux et programme de stock $^{16}$, à cause de son hybridité. S'agit-il vraiment d'une fiction? Du côté du spectateur, quelles formes de l'audiovisuel sont identifiées facilement comme appartenant au «réel»? Ensuite l'effet produit par la reconnaissance de codes journalistiques dans une fiction semble paradoxal : si le style du fait divers est là pour "faire vrai ", l'artificialité de son usage, le décalage avec le contenu, sans volonté parodique, est problématique. Enfin, qu'est-ce qui concrètement justifie l'émergence de ce genre hybride dans la grille des programmes de télévision française? Quels sont les enjeux de cette importation?

\section{Fiction ou réel : quelle promesse faite aux spectateurs?}

Quel pacte, ou quelle " promesse $^{17}$ » selon le terme de François Jost relie le programme aux spectateurs? Le générique de Si près de chez vous est extrêmement court et se scinde en deux parties. Sur fond bleu clair, neutre, accompagnée par quelques notes allant crescendo et pouvant rappeler d'autres génériques de magazines de société, la mention «cette histoire est basée sur un fait divers réel » s'incruste à l'écran sur une ligne centrale. Le bruit reconnaissable d'une machine à écrire se superpose à l'apparition de ce message, évoquant alors l'atmosphère des salles de rédaction des journaux, ce qui réoriente la première impression vers le genre de l'enquête, du mystère. En outre la formule est bien connue des amateurs du genre policier : beaucoup de ces fictions se déclarent inspirées de faits réels. Ces quelques secondes lancent la séquence prégénérique qui s'ouvre effectivement sur la reconstitution d'un crime. Arrive alors la seconde partie du générique. Le fond bleu réapparaît, sur lequel un bouquet de noms de villes françaises écrites en noir et blanc jaillit : Paris, Dijon, Montpellier, Valence... ce chapelet de toponymes crée un premier lien de proximité avec le spectateur qui aperçoit nécessairement un lieu connu. Outre la proximité, c'est aussi la dimension aléatoire qui est ainsi mise en scène dès les premiers instants par ces apparitions fugaces durant lesquelles des noms de ville se détachent par un jeu d'échelle et de couleur : cela peut évoquer un tirage au sort, un jeu de hasard. Quelques notes allant 
cette fois-ci decrescendo closent le générique, avec lors des dernières secondes l'apparition du titre: Si près de chez vous, traçant la même ligne que le premier message.

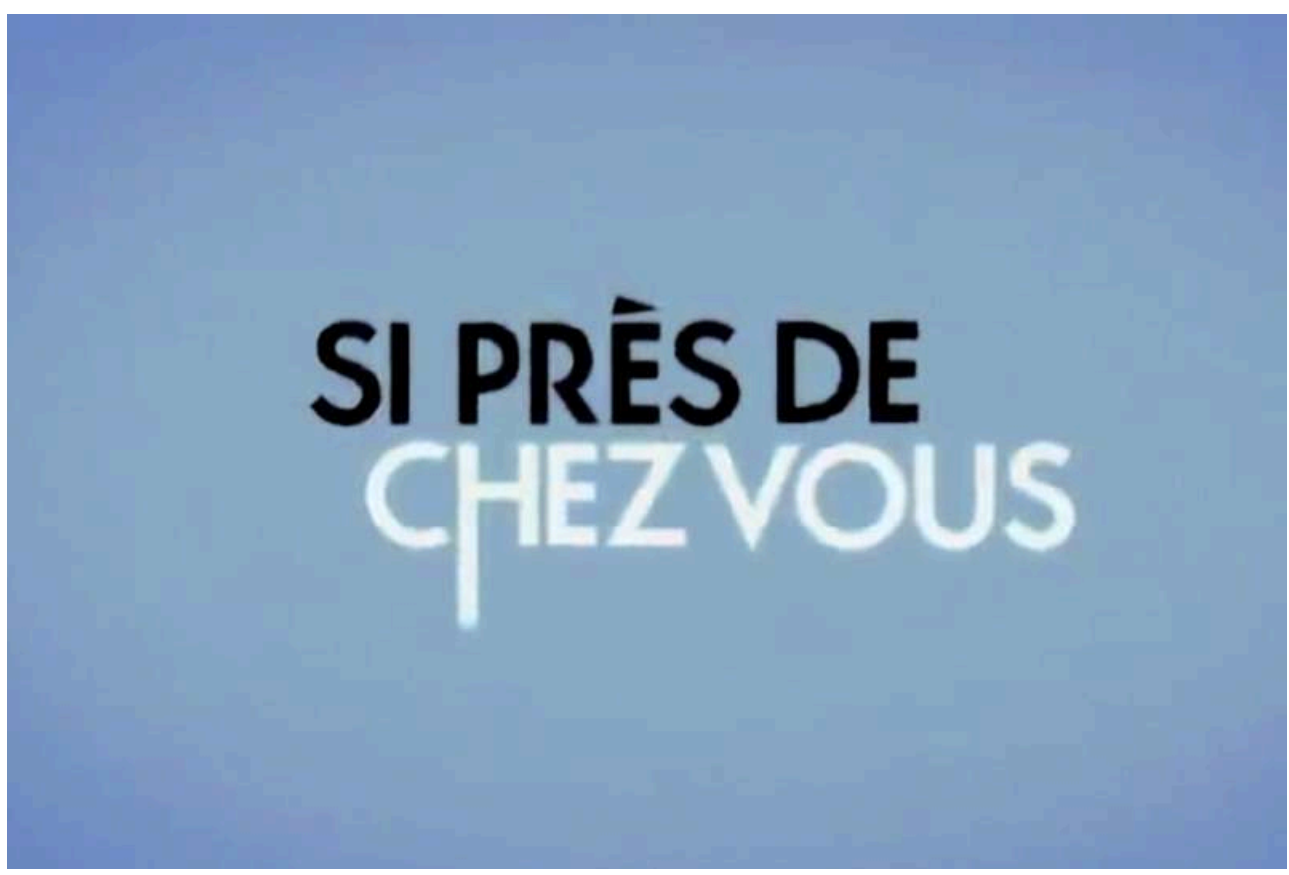

(Photogramme : 909 Productions, France Télévision)

7 Ce titre joue sur l'un des aspects essentiels du fait divers : la proximité. Le fait divers touche les gens parce qu'ils peuvent s'identifier, que les raisons soient géographiques, socio-culturelles, socio-professionnelles ou personnelles (âge, sexe, rôle familial). Par l'adverbe intensif, ce titre insiste d'emblée sur la préposition clé "près de ", comme pour rendre explicite l'idée latente que cela peut arriver à tout le monde et que cela aurait pu arriver à celui qui regarde l'émission. Pas de verbe justement, et pour une raison simple : le titre évoque immédiatement pour les amateurs de faits divers le titre $\mathrm{du}$ film noir C'est arrivé près de chez vous ${ }^{18}$, jouant donc ici ironiquement de la variation intertextuelle. Sans même connaître la référence, on peut dire que le titre est suffisamment repris dans les médias et passé presque comme une expression lexicalisée pour que la variation soit identifiée. La valeur rhématique ici du syntagme prépositionnel est autosuffisante. Mais l'adverbe "si » laisse peut-être entendre également, par un processus de double énonciation, l'éternel étonnement des témoins de drames du quotidien qui s'expriment à grand renfort d'hyperboles et n'en reviennent pas que cela se soit passé « juste » là, « tout » près, « si » près... Enfin, il est porteur de la menace inhérente à tout fait divers. Il permet d'ouvrir les yeux sur la dangerosité du monde a priori si normal et banal.

8 Tout en s'adressant directement au spectateur, le titre permet aussi de situer le programme dans l'ordre de l'intime : «chez vous », est une localisation géographique mais évoque également le "chez soi ». Le titre annonce l'irruption de l'événement inattendu au cœur du journalier, du quotidien, et le risque de voir sa vie basculer (qui est le titre d'une autre fiction de scripted reality d'une chaîne concurrente).

Chaque épisode est également pourvu d'un titre, qui apparaît discrètement lors du générique en bas, à droite de l'écran, et reste affiché pendant les vingt-six minutes. Sa forme brève ("Destins croisés », «Mauvais frère »), parfois informative («Un enfant vendu sur internet », « Meurtre d'un riche héritier », « Un divorce qui tourne mal »), sa 
tendance à la stéréotypie ("Vengeance», "Le Cœur et l'argent»), au métadiscours médiatique ("Mort à la une », "Photos choc») et à la littérarisation ("Roman noir », «Le Mystère de l'auberge », « De sang-froid ", « Randonnée mortelle »), évoquent bien sûr la titraille classique du petit fait divers ${ }^{19}$.

La séquence inaugurale de chaque épisode est en quelque sorte la confirmation de ce titre, tout en opérant le passage du général au particulier, par la thématisation d'un lieu (région, ville) et d'une scène (appartement, maison) :

En Normandie, le haras le plus réputé de la région est le théâtre d'une agression sans précédent.

Dans un appartement parisien, un homme blessé à la gorge, se trouve entre la vie et la mort. Non loin de lui, un couteau ensanglanté.

Un appartement dans le $8^{e}$ arrondissement de la ville de Marseille. Un homme git sur le sol, la chemise couverte de sang ${ }^{20}$.

On observe une économie de moyens pour créer le cadre référentiel : que cela soit par un groupe prépositionnel en tête de phrase (« en Normandie »), un adjectif relationnel (" parisien ») ou une phrase averbale, la scène est ébauchée rapidement, évoquant la brièveté du petit fait divers. Cette séquence pré-générique dure en moyenne 45 secondes. La particularisation de l'épisode commence donc par une géolocalisation, même si parfois certains épisodes ne mentionnent pas immédiatement cette information. Ainsi de :

Julien, un gendarme de 44 ans, est retrouvé entre la vie et la mort dans un jardin du village où il habite.

Albin, 45 ans, metteur en scène talentueux vient d'être abattu de sang-froid sur la scène de son théâtre $e^{21}$.

Pour ces deux épisodes, ce n'est qu'après le générique que l'on peut situer géographiquement la scène du crime, l'un dans « la région des Ardennes ", l'autre dans « la région de Grenoble ». L'entrée ici se fait par la victime. La nomination crée un effet de proximité très fort et immédiat, tandis que les informations portant sur l'âge et la profession permettent au spectateur de se situer par rapport à cette victime. La cible visée étant traditionnellement la ménagère de moins de 50 ans, on observe que la victime, masculine ou féminine, entre souvent dans la catégorie des quadragénaires. La seule mention du prénom rappelle également l'anonymat de rigueur dans la rubrique fait divers des quotidiens. De manière générale, dans cette séquence introductive, l'apposition permet une présentation rapide de la victime : «Sa propriétaire, Charlotte, une femme de poigne, est violemment attaquée ${ }^{22}$.» Plus rarement ces informations sont distillées au fur et à mesure du paragraphe, ménageant une gradation :

Un appartement dans le $8^{\mathrm{e}}$ arrondissement de la ville de Marseille. Un homme gît sur le sol, la chemise couverte de sang. Sébastien vient d'être assassiné, deux balles dans l'estomac, tirées à bout portant. Que s'est-il passé ? S'agit-il d'un règlement de compte? Est-ce un cambriolage qui a mal tourné ? Ou un crime passionnel ? Le romancier à succès de quarante ans avait-il des ennemis capables d'attenter à ses jours ${ }^{23}$ ?

13 La chaîne anaphorique permet une découverte progressive du personnage de la victime : le groupe nominal indéfini est repris par le prénom, puis l'anaphore infidèle permet de préciser les contours de cette victime. La caractérisation n'est pas systématique et souvent discrète, observant une certaine neutralité. Cependant, la moindre épithète oriente déjà la suite du récit vers ce qui sera au cœur de l'épisode : les motifs du crime. Ainsi un metteur en scène « talentueux », un romancier « à succès » ou une femme "de poigne", dans ce contexte de réception - un fait divers réel -, 
éveillent immédiatement les soupçons du spectateur. Ces quelques indices sont rendus explicites par la clôture de la séquence sous la forme d'une série de questions semifermées posées au spectateur. Cette première séquence inaugurale se fait donc explicitement sous le patronage du fait divers. Outre la mention de départ, on reconnait l'efficacité, la neutralité, du cadre référentiel recréé en quelques secondes.

Cependant la promesse faite au téléspectateur n'est pas claire: faut-il prendre «histoire » au sens de fiction? S'agit-il d'une reconstitution d'un fait divers présenté sous la forme d'une enquête rétrospective comme dans un magazine? Va-t-on voir des témoins en plateau pour raconter cette histoire vraie ? Le public doit se fier à sa « media literacy» selon le terme d'Isabelle Veyrat-Masson ${ }^{24}$, même si celle-ci est parfois trompeuse : reconnaître par exemple la voix d'Alain Ghazal, récurrente dans Si près de chez vous, n'aidera pas à classer l'émission, ce dernier travaillant aussi bien pour des magazines (Chroniques criminelles) que des documentaires (Arte). Cette séquence dispense des indices contradictoires qui permettent difficilement de trancher ${ }^{25}$.

Cette indistinction est renforcée par une absence de mention de genre, aussi bien sur l'œuvre même que dans le péritexte. Les programmes TV ne s'accordent pas sur la caractérisation de l'émission. Pour Télérama, Télé deux semaines ou Télé-loisirs, Si près de chez vous est un magazine de société, alors que Téléstar précise: "genre: Série/ Feuilleton - Documentaire fiction. » TV magazine le classe dans la même catégorie en ajoutant ce commentaire : « un polar du réel qui s'inspire d'histoires vraies qui se sont passées près de chez nous ». Il s'agirait d'une série policière. Les programmes télé n'utilisent donc pas le terme récemment importé de scripted reality, mais tentent de faire entrer l'émission dans des cases préexistantes.

L'arrivée de la scripted reality à la télévision française suscite aussi un débat auprès des instances de l'audiovisuel, largement relayé dans les médias ${ }^{26}$. Le CSA se prononce en ces termes :

L'évolution récente des émissions montre une hybridation de plus en plus fréquente entre plusieurs genres traditionnels de programmes. Celle-ci a pour conséquence de rendre moins évident le rattachement de ces émissions à l'une ou l'autre des grandes catégories de programmes ${ }^{27}$.

17 À l'issue de cette enquête, le CSA octroie le statut de fiction à Si près de chez vous. En revanche pour le Centre National du Cinéma et de l'image animée (CNC), les émissions de réalité scénarisée n'entrent pas dans les critères des œuvres patrimoniales, ne sont pas des fictions, et ne bénéficient pas de subventions ${ }^{28}$. Cette hésitation sur le statut juridique du programme est donc une conséquence de son hybridation formelle.

\section{Hybridation}

Les débats qui se sont tenus en 2013 pour établir juridiquement le genre de ces émissions s'expliquent par leur forme hétéroclite. Dans Si près de chez vous, outre un lancement qui évoque le traitement fait-diversier du journal télévisé, on retrouve les modèles du magazine de faits divers, du reportage et du magazine de société, sans oublier sa structure standardisée et répétitive qui l'apparente à une série. 


\section{Avec le magazine de fait divers}

19 La séquence pré-générique instaure immédiatement la thématique criminelle. Chaque épisode présente ainsi un lexique de l'homicide (« assassiné, deux balles dans l'estomac, tirées à bout portant ", " agression sans précédent ", " blessé à la gorge ", " retrouvé entre la vie et la mort») ou une caractérisation hyperbolique, à la manière du fait divers d'actualité.

En revanche ce qui marque une rupture très franche avec ce dernier est le moment de l'énonciation: alors que dans la presse ou au journal télévisé le journaliste narrant évoque un fait passé proche, mais révolu, à partir du présent de la publication - souvent grâce au passé composé -, ici la voix off hors champ qui prend en charge la narration s'ancre dans le présent du crime, ou dans le passé immédiat de ce dernier : le moment de l'énonciation semble être quasi concomitant à celui du crime, d'où l'usage du présent, à valeur d'actualité, ou très fréquemment de la périphrase verbale «venir de » indiquant un passé proche :

Dans un appartement parisien, un homme blessé à la gorge, se trouve entre la vie et la mort. Non loin de lui, un couteau ensanglanté.

Un appartement dans le $8^{\mathrm{e}}$ arrondissement de la ville de Marseille. Un homme gît sur le sol, la chemise couverte de sang. Sébastien vient d'être assassiné, deux balles dans l'estomac, tirées à bout portant.

Albin, 45 ans, metteur en scène talentueux vient d'être abattu de sang froid sur la scène de son théâtre.

En Normandie, le haras le plus réputé de la région est le théâtre d'une agression sans précédent. Sa propriétaire, Charlotte, une femme de poigne, est violemment attaquée. À côté de son corps, se trouve une statue ensanglantée, probablement l'arme du crime $^{29}$.

21 Si l'emploi du présent avec un verbe imperfectif comme "se trouver» peut se rapprocher du présent d'énonciation tel qu'on le trouve dans la presse, en revanche son entourage linguistique empêche la confusion. Là où le fait divers ne peut que suggérer, arrivant nécessairement trop tard sur les lieux du crime, Si près de chez vous introduit le spectateur au moment clé. Se superposent alors le moment du vrai crime et celui où on le rejoue, le programme jouant de la confusion entre trois temps : celui, éloigné, des faits, celui plus récent du tournage et enfin le présent ponctuel de l'image. À l'écran, après un cadrage large sur la scène de crime, l'attention du spectateur est portée sur le corps de la victime, allongée, sanglante, inerte. Parfois même l'agression est montrée en caméra subjective, masquant ainsi l'identité du criminel mais montrant la mort de la victime. Le présent permet donc de commenter en temps réel l'image. Chaque épisode commence par un début in medias res, s'éloignant ainsi de l'écriture journalistique. 


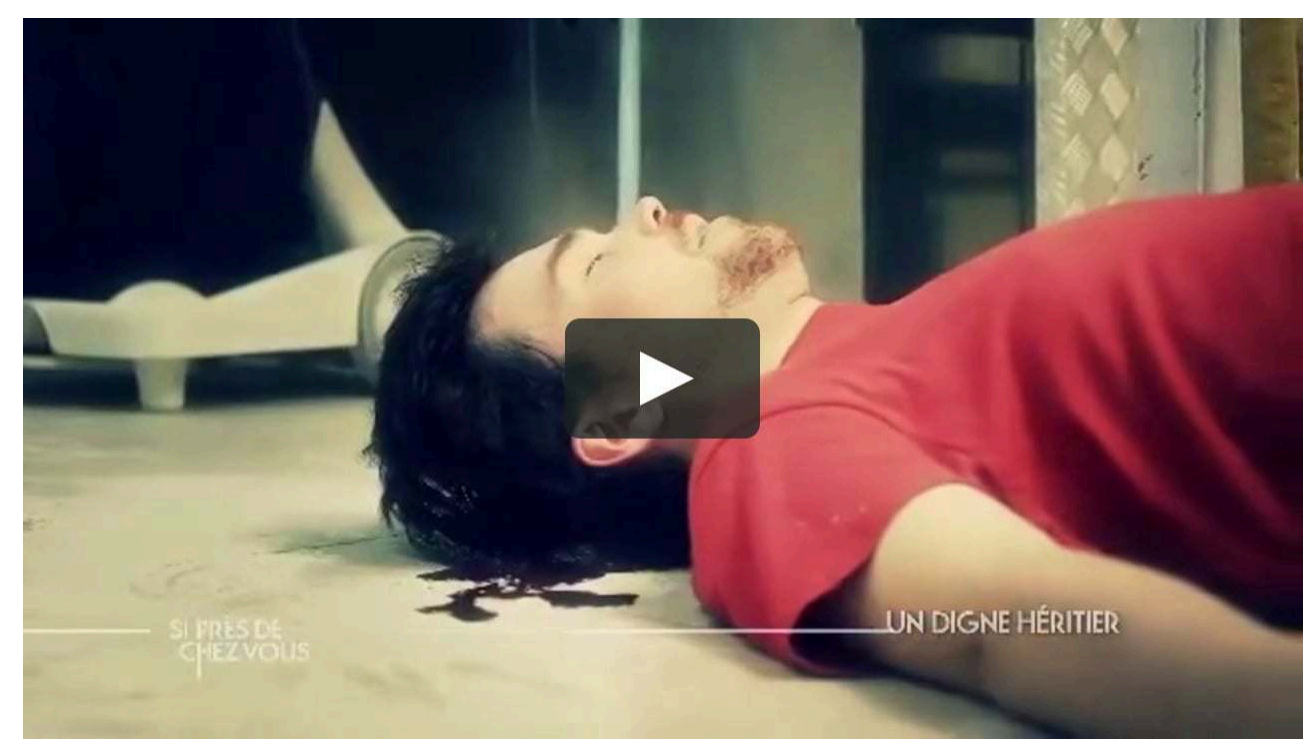

« UN DIgNE HÉRITIER », SI PRÈs DE CHEZ VOUS, 2012.

(PHOTOgRAMME : 909 PROdUCtIONS, FRANCE TÉLÉVISION)

Cependant une telle représentation du crime ne bouleverse pas outre mesure le spectateur habitué à la médiatisation du fait divers criminel. En effet, si l'on quitte le genre d'actualité rencontré dans le journal télévisé, on retrouve un autre genre, consacré au crime : le magazine.

Faites entrer l'accusé sur France 2, Enquêtes criminelles sur W9, Suspect $n^{\circ} 1$ sur TMC, Présumé innocent sur D8, Enquête de vérité sur NRJ 12 témoignent de l'intérêt que portent les chaînes aux émissions spécialement consacrées aux faits divers qui occupent généralement une place de choix dans leur grille de programmation : $1^{\text {re }}$ ou $2^{\mathrm{e}}$ partie de soirée ${ }^{30}$.

Alors que le fait divers au journal télévisé fait partie de l'actualité, ce type de programme propose de se replonger dans une affaire plus ou moins ancienne $a$ posteriori. L'une des premières séquences de ces magazines est la découverte du corps, reconstituée grâce au jeu d'un comédien qui tient le rôle de la victime et racontée en voix off.

Les journalistes disposent d'un temps de conception beaucoup plus important, certains artifices sont alors possibles comme la reconstitution du drame par des acteurs. Le téléspectateur peut ainsi voir un comédien tirer un coup de feu sur un autre jouant le rôle de la victime, ou des scènes de la vie quotidienne qui ont précédé les faits et permettent de comprendre les motifs du crime. Procédant à une forme de fictionnalisation $\mathrm{du}$ fait divers par ces images, les émissions hebdomadaires consacrent également un temps plus important à la narration de l'événement. La durée du reportage est le deuxième point distinguant le format $d u$ fait divers au JT à celui de ces émissions ${ }^{31}$.

Le regard porté sur le corps est celui du témoin qui a découvert le crime, généralement un proche. La caméra se porte sur la victime sans vie en focalisation interne, le témoin réel ne participant pas à la reconstitution, tandis que la voix off raconte. Souvent cette dernière est en revanche relayée par le témoin qui, face caméra, raconte avec ses propres mots la découverte macabre.

25 Le dispositif qui se met en place dans Si près de chez vous est assez similaire, à un détail près : l'absence de témoin. Si la caméra donne accès au corps sans vie, on ne sait pas qui regarde. Le spectateur se retrouve donc dans le rôle de témoin $n^{\circ} 1$, au côté de ce 
narrateur qui l'a introduit sur les lieux du crime. On peut également s'interroger sur la voix présentant la scène: alors que dans les magazines de faits divers, la scène énonciative englobante est clairement établie à l'ouverture, par la présence d'un journaliste et/ou d'experts, ici elle reste extrêmement ambiguë puisque la reconstitution ne dit pas son nom et que le seul regard qui se pose sur la victime est celui d'un narrateur omniscient. Dans le magazine, tantôt un journaliste, tantôt un expert, endosse la responsabilité du récit. De même les reconstitutions sont exhibées comme telles grâce à un petit encart à l'écran. Dans la scripted reality, certains codes énonciatifs du magazine, sont repris, mais gratuitement, pour la forme.

Enfin, comme dans ces magazines de fait divers qui empruntent leur mode de narration au reportage écrit, on retrouve le principe organisateur de l'enquête puisqu'il s'agit de savoir qui a commis le crime. "Véritable polar du réel, "Si Près De Chez Vous" vous entraîne au cœur des faits divers... Chaque jour sur France 3 et $13^{\text {ème }}$ Rue, c'est vous qui menez l'enquête! » explique 909 Production sur son site. La reconstitution initiale, dans Si près de chez vous, sert de point de départ au déroulement de la fiction. À partir de cette scène post-criminelle, la narration procède à une longue analepse, signalée très souvent par une phrase averbale du type "retour sur»: un point $B$ antérieur à ce point $\mathrm{A}$ est posé, à partir duquel le récit se déploie de manière linéaire jusqu'à revenir au moment du crime. Cependant la narration s'affranchit de son modèle journalistique par plusieurs aspects : il n'y a pas d'enquêteur, si ce n'est le spectateur ; il n'y a pas d'investigations puisque l'on se situe avant l'acte.

Revenons six mois plus tôt, dans cette petite bourgade située à deux pas du Havre.

C'est dans le $13^{\mathrm{e}}$ arrondissement de la capitale que le drame s'est noué quatre semaines plus tôt.

Dans un petit village bordelais 3 mois plus tôt.

Tout a commencé au cœur de la cité phocéenne il y a quelques semaines ${ }^{32}$.

Le spectateur retrouve le cadre spatial et le personnage présentés dans le prégénérique, avant qu'ils ne soient associés à un crime. Pourtant celui-ci ne peut pas s'oublier: ce qui oriente le récit c'est donc le "qui» et le "pourquoi», que le spectateur, mis en position d'observateur extérieur, va tâcher de découvrir. Le récit est raconté au présent et montre le quotidien de la future victime. Cependant pour ne pas perdre de vue l'issue funeste, des interventions proleptiques de la voix off rythment l'épisode :

Dans quelques minutes cette dernière sera violemment frappée à la tête (24:35).

Nouvelle histoire sentimentale, bel appartement, tout semble réussir au jeune informaticien. Et pourtant son destin va bientôt basculer tragiquement. Comment la situation a-t-elle pu dégénérer au point d'en arriver là? Dans moins de 24 heures, Laurent sera retrouvé gisant sur le sol de sa cuisine, la gorge tranchée (20:05) ${ }^{33}$.

Ces transitions interviennent entre chaque séquence, établissant un bilan, rappelant l'enjeu à venir, et orientant les spectateurs par des questions sur l'identité du criminel en lien avec un mobile. Dans les magazines de faits divers, ces éléments sont utilisés pour souligner un moment fort, par exemple la découverte d'un nouvel indice ou un retournement de situation. Une rhétorique d'effets spectaculaires est alors utilisée, reprise dans Si près de chez vous : images avec surimposition de filtres de couleur, slow motion, notes de violon lancinantes et répétitives créant un effet anxiogène et pathétique, gong final, avec effet d'écho qui marque la fin de la séquence et l'ouverture de la nouvelle. Encore une fois la réalisation de Si près de chez vous compte sur la reconnaissance de ces effets du magazine, tout en les détournant de leur emploi initial. 
Ils ne sont pas là pour mettre en relief un twist, mais pour rythmer le récit, créer une tension au milieu de la narration, qui s'apparente sans cela à un autre genre médiatique : le magazine de société.

\section{Avec le magazine de société}

Le magazine de faits divers mène donc une enquête rétrospective pour percer le mystère d'un crime. Plusieurs instances légitimes interviennent dans cette quête: police, détective privé, avocat, et reporter spécialisé, ce qui n'est pas le cas de Si près de chez vous. Chaque épisode se présente comme une reconstitution du quotidien le plus banal de la victime avant le drame. Ce récit de vie s'effectue dans une alternance de trois modes :

- Voix off (récit), extérieure, omnisciente, encadrante, elle a une fonction d'explicitation de l'image et de jointure entre les scènes.

- Scènes (mimesis), conversations, menus faits du quotidien. Le spectateur observe la vie des personnages.

- Témoignages de protagonistes en plans fixes face à la caméra avec incrustation de mentions à l'écran donnant des indications sur leur identité.

Ce dispositif est très fréquent depuis une quinzaine d'années à la télévision : c'est celui que l'on retrouve dans toutes les émissions de téléréalité, et que les magazines de société et de faits divers ont adopté et adapté. Comme le notent Patrick Baudry, Claude Sorbets et André Vitalis, l'intime, la vie privée, sont devenus des sujets attractifs, s'accompagnant d'une "libération de la parole ordinaire ", au détriment de celle des experts :

La place qu'occupe la vie privée dans les médias et notamment à la télé correspond sans doute à une stratégie communicationnelle. Il s'agit de jouer de la proximité, de la projection et de l'identification : le monde des médias ne serait pas différent du monde vécu, il serait ce monde même. Entre le spectateur et ce qu'il voit, il faudrait abolir la distance du spectacle : il faudrait que la mise en spectacle de la réalité apparaisse comme le réel même ${ }^{34}$.

31 La narration hors champ relie les scènes dialoguées par des sommaires accélérant le rythme interne. Elle instaure la tension narrative en gardant en ligne de mire le crime futur, alors que les échanges dialogués entre les personnages permettent de plonger au cœur du quotidien, rappelant le « culte du banal » selon Jost. Le temps semble alors extensif (hors tension). Si près de chez vous met en scène la même trivialité que dans le magazine de société ou dans la téléréalité où le spectateur s'invite chez les gens : petitdéjeuner, rhume, vaisselle, factures à payer, réunion à l'école, le quotidien est mis en scène. Chaque épisode commence par une scène d'une extrême banalité, introduite par la voix off :

Cela fait seulement quelques mois que Laurent et Adeline se sont rencontrés, un véritable coup de foudre, et depuis ils font déjà de grands projets. Ce jour-là, Adeline découvre l'appartement de celui chez qui elle va s'installer bientôt. (1:11)

LAURENT : « Et voilà !

ADELINE : Wouah! Mais c'est génial!

LAURENT : Ça te plait?

ADELINE : Ouais! C'est somptueux chez toi !

LAURENT : Je l'ai acheté cette année !

ADELINE : Eh ben ! ça rapporte les boîtes informatiques !

LAURENT : Ah ben ça je te l'ai toujours dit, hein! 
ADELINE : Dire que tu as mis deux mois avant de me montrer où tu vivais...

LAURENT : Ah oui mais c'était en plein travaux... J'ai refait toutes les peintures là... Mais maintenant... il va falloir un peu d'espace... (Il pose la main sur le ventre de la jeune femme)

ADELINE : Arrête de parler comme ça, ça porte malheur, ça fait qu'un mois et demi, rien n'est sûr...

LAURENT : Oui je sais, je sais, il faut attendre la première échographie... [...]

ADELINE : Waouh, on va être bien ici $^{35}$... »

Certains titres d'épisodes semblent aussi faire écho au magazine de société, en utilisant des déterminants possessifs ou des pronoms personnels qui permettent de placer un individu lambda au centre de l'intérêt : « Mon ex me harcèle ", «Indigne de ma fille »; «Mariage: le pire jour de sa vie»; "Une femme trop belle pour lui »; «Je ne me souviens de rien $^{36} »$.

Ces scènes ne sont pas seulement jouées par des comédiens: elles sont également commentées. Court-circuitant la diégèse, la réalisation insère des témoignages en plan fixe, dans un non-lieu qui reprend le même fond bleu que le générique. Ces codes sont empruntés à la téléréalité et au magazine de société type C'est mon choix, Confessions intimes, Toute une histoire, Tellement vrai, faisant alterner tranche de vie et retour des protagonistes sur les moments montrés, généralement exprimés au présent.

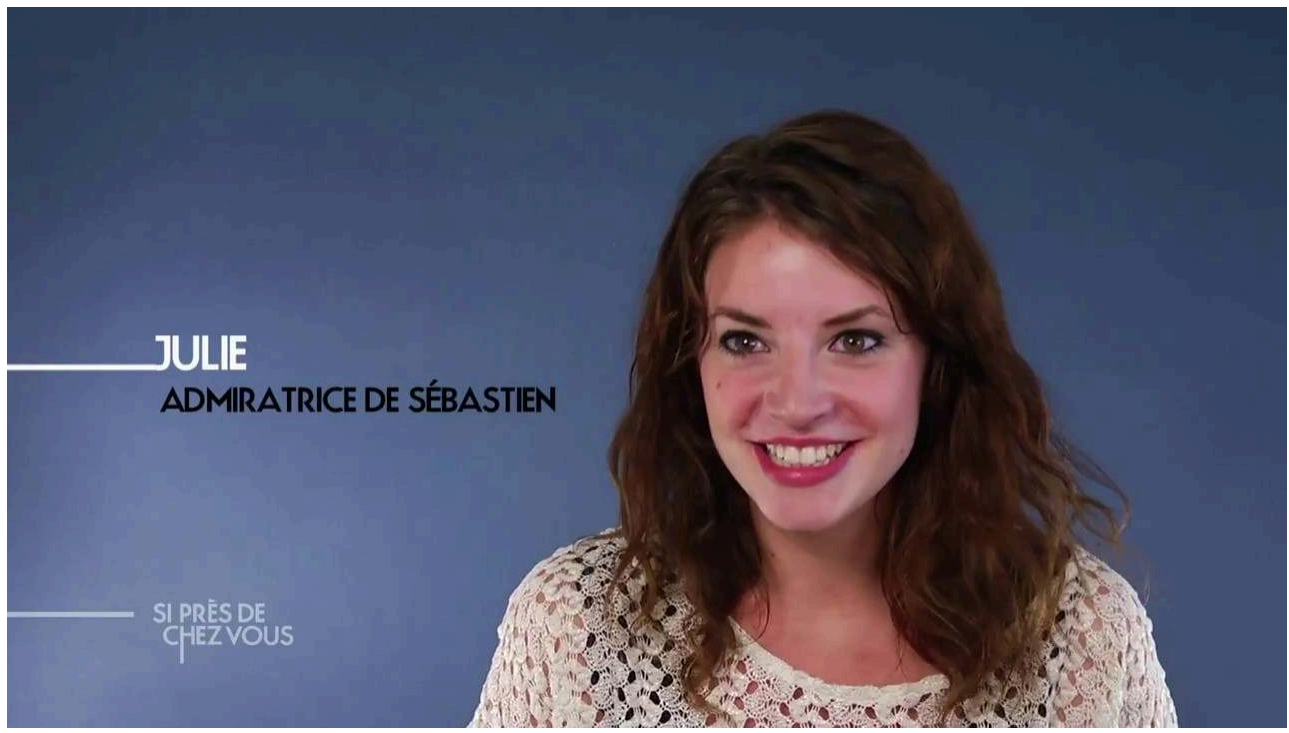

"L'Homme derrière les mots », Si près de chez vous, 2012.

(Photogramme : 909 Productions, France Télévision)

Sur ce même modèle dans Si près de chez vous, après chaque scène, un témoignage face caméra vient ponctuer l'avancée du récit :

ADELINE: «C'est sûr, j'étais pas habituée à un tel luxe. [...] Là il faut reconnaitre qu'il

savait me faire rêver Laurent. C'est sûr que d'élever son enfant dans un environnement comme celui-là, c'était plutôt rassurant. »

VICTOR : «C'est vrai que j'étais impatient, je voulais tellement que ça marche !»

KIM : «C'est vrai que j'étais épuisé, que j'avais de la fièvre ${ }^{37}$. »

35 Au niveau de la narration, ces témoignages permettent d'approfondir les portraits factuels et psychologiques des personnages sans recourir à la voix off. La modalité aléthique très présente, de même que l'emploi de déictiques, semblent montrer que les personnages commentent la reconstitution scène après scène, dans une situation d'énonciation postérieure au drame. L'imparfait est le temps dominant. Ces trois 
personnages deviennent donc des relais du narrateur principal, mais instaurent une narration ambiguë : alors qu'ils connaissent l'issue, - l'un d'entre eux étant même le coupable, - chaque commentaire s'en tient au moment montré à l'image, maintenant ainsi une focalisation interne sur les événements. Ce procédé, qui n'est pas sans rappeler par exemple Le Meurtre de Roger Ackroyd ${ }^{38}$, permet ponctuellement de tromper le spectateur en l'embarquant sur de fausses pistes, notamment par l'extrême modalisation des discours. Ainsi les témoignages sont très souvent emphatiques, exprimant la colère, la haine, la douleur :

AMÉLIE : « Si seulement on avait fait opérer le cheval de papa comme l'avait conseillé Alain... Mais non, elle, elle préférait changer de gravier! Elle devenait folle ou quoi? On n'était vraiment plus sur la même longueur d'onde. »

ALAIN : " [...] Mais enfin c'est pas comme ça qu'on traite les gens! Je m'en voulais de ne pas avoir compris plus tôt ce qu'il se passait ; mais maintenant j'ouvrais l'œil, et elle avait intérêt à faire le nécessaire parce que moi, j'allais pas abandonner ${ }^{39}$. »

Alors que ces témoignages sont effectués sur le même fond bleu ciel neutre que le générique, avec un plan poitrine, le comédien rejoue presque la scène, notamment par l'intonation de voix, le débit, ou même certaines formulations ambiguës. Ainsi la phrase exclamative d'Alain semble être du discours direct libre, qui laisse entendre à la fois une phrase que le personnage aurait pu prononcer au moment des faits et l'indignation qu'il rejoue au moment de les raconter. De même la formulation de la question « elle devenait folle ou quoi? » semble hésiter entre d'une part l'interpellation orale directe et le récit. On aurait donc ici du discours indirect libre, le personnage d'Amélie reformulant une altercation qu'elle aurait eue avec sa belle-mère. Ces hésitations s'entendent aussi parfois dans la confusion énonciative mêlant discours ancré et coupé, avec par exemple l'usage du déictique «maintenant» associé à l'imparfait «j'ouvrais l'œil » qui crée la confusion. Parfois c'est tout un témoignage qui balance entre impressions à vif et rétrospection :

VICTOR : "J'étais écœuré, je me suis senti trahi : comment il a pu oser ? Son succès il me le devait, tout ça c'était grâce à moi. Pendant des années j'ai été son nègre, je répondais même au courrier des lecteurs! J'ai toujours accepté cette situation mais je rêvais d'autre chose et il le savait. Comment il a pu se permettre de me faire ça! (Cri de rage) ${ }^{40}$."

Le récit à l'imparfait laisse ici place au passé composé qui est majoritaire. Victor ne semble plus parler depuis un temps coupé de la diégèse, permettant le commentaire, mais relié à celle-ci, afin d'exprimer ce qu'il ressent juste après la scène visionnée. Au niveau de la narration, ces témoignages ont donc pour but d'entretenir le suspense quant à l'auteur du crime. Ces témoignages se concluent ainsi souvent par des menaces, formulées grâce à un futur dans le passé qui laisse planer les soupçons sur l'énonciateur :

$\mathrm{KIM}:$ « Il allait le payer cher, très cher... »

AMÉLIE : « Je commençais sérieusement à la prendre en grippe. »

ALAIN : «Et si elle allait trop loin, elle allait le regretter ! [...] Et si Kim foutait le

camp, ça lui donnerait une bonne leçon ${ }^{41}$. »

Le dispositif narratif mis en place dans cette émission de réalité scénarisée est donc complexe : à la voix off omnisciente, qui régit le tempo et la tension narrative, s'ajoutent celles des narrateurs internes, sous la forme de témoignages oscillant entre confidences rétrospectives et commentaires à chaud permettant de maintenir le suspense quant au criminel. Or le spectateur sait que ce dernier est l'un d'entre eux, ce qui permet de miner de l'intérieur le dispositif issu des émissions de téléréalité puisque 
ces témoignages ne sont pas fiables. Ou plutôt, selon le procédé de la paralipse, on sait que cette narration chorale dissimule des informations sans vraiment mentir. Le spectateur reconnaît bien là un mode informatif d'énonciation, mais il est mis au service de la fiction ${ }^{42}$.

Ce n'est que dans la dernière série de témoignages, après la scène de crime qui dévoile l'identité du meurtrier, que le temps du témoignage rejoint celui de la diégèse et que la connaissance des faits du spectateur devient identique à celle des personnagestémoins. La présence de ces témoignages face caméra est donc un pastiche d'une forme non fictionnelle que l'on rencontre dans les magazines de société ou de fait divers. Facilement identifiables, ils permettent de "faire vrai », tout comme la mention « inspiré d'un fait divers réel », mais servent en réalité à construire un récit complexe.

\section{Sérialisation et enjeux}

40 À l'issue de ce parcours, on peut donc dire que Si près de chez vous est une fiction du réel qui joue sur des codes génériques télévisuels pour se donner les apparences du vrai ${ }^{43}$. Il est cependant indispensable de relier cette médiapoétique au contexte de production qui l'a engendrée, ainsi résumé dans le compte rendu du CSA :

Les éditeurs et les producteurs directement intéressés insistent sur la nécessité économique de trouver en journée des programmes nouveaux, identifiants (car il y a saturation de rediffusions), qui puissent être produits à des prix comparables à ceux des programmes de flux ou des achats de droits de second marché [... $]^{44}$.

41 L'importation de la scripted reality est avant tout guidée par un besoin : remplir la grille des programmes de journée sur les tranches horaires $10 \mathrm{~h}-12 \mathrm{~h}$ et $14 \mathrm{~h}-16 \mathrm{~h}$, traditionnellement occupées par des feuilletons américains (Les Feux de l'amour pour TF1) ou des magazines de société (Toute une histoire pour France 2). La contrainte économique est forte : il faut produire à bas coût, puisque l'audience est faible. Or la scripted reality en 2012 apparaît comme une stratégie gagnante pour les différentes chaînes, puisqu'elle permet une certaine continuité avec les programmes habituels - thématique et formelle, comme vu précédemment -, tout en les renouvelant. Pour les professionnels «inquiets à la fois de n'être pas trop innovants et inquiets de ne pas l'être asse $\mathrm{z}^{45}$ » chargés de la programmation, elle présente certains avantages.

D'un point de vue technique, les conditions de production de Si près de chez vous sont proches de celles des émissions de flux. C'est ce que produit majoritairement 909 Productions. Seule fiction de la société, la série bénéficie de la même organisation industrielle destinée à réduire le coût et le délai de fabrication. Ce mode de production transparaît dans la standardisation des épisodes, tous écrits selon un script identique.

Le casting de chaque épisode est ainsi resserré à quatre personnages. La sérialisation - plus précisément, l'effet «formule ${ }^{46}$ »- permet rapidement de savoir que le coupable est l'un des trois personnages qui entourent la future victime. Cette standardisation se lit dans les synopsis des programmes TV. Celui du premier épisode paraît emblématique :

Dans les Pyrénées-Orientales, Laurent, 40 ans, vétérinaire et défenseur de la cause animale, est retrouvé mort, tué de deux balles tirées à bout portant. Trois personnes de son entourage sont entendues par la police ${ }^{47}$. 


\section{spectateur-enquêteur tâche de relier aux trois nouveaux personnages qu'on lui soumet :}

Que s'est-il passé ? S'agit-il d'un règlement de compte ? Est-ce un cambriolage qui a mal tourné ? Ou un crime passionnel ? Le romancier à succès de quarante ans avaitil des ennemis capables d'attenter à ses jours ${ }^{48}$ ?

Après une première question ouverte et générale, la voix off propose plusieurs pistes précises (règlement de compte, cambriolage, crime passionnel). La dernière question sur l'existence hypothétique « des ennemis » de la victime introduit les trois suspects qui sont ici l'ex-femme de l'écrivain, son porte-plume et ami, et une fan. La répétition d'un même script d'épisode en épisode permet de savoir qu'il n'y aura pas de deus ex machina : pas de criminel sorti de nulle part, c'est à chaque fois un petit huis clos qui se déroule ${ }^{49}$.

Enfin la standardisation apparaît aussi bien au niveau narratif, chaque épisode se construit sur un canevas très serré, qu'au niveau des personnages qui sont tous stéréotypés. La mise en série à partir d'un script fixe de ces petits faits divers permet donc de remplir la grille de journée pour un prix aussi bas que pour une émission de flux $^{50}$, mais avec un bénéfice supplémentaire par rapport à celle-ci : "répondre aux obligations de production d'œuvres patrimoniales ». En effet, les chaînes doivent respecter «les quotas de diffusion d'œuvres européennes et d'expression originale française » et ont des « obligations de contribution au développement de la production audiovisuelle européenne et d'expression originale française » (entre 11,5\% et $20 \%$ des investissements, selon les chaînes, calculés sur les bénéfices de l'année qui précède). Comme le résume bien le rapport du CSA :

L'enjeu de la qualification en fiction des programmes de "réalité scénarisée » réside donc dans leur prise en compte dans les obligations d'investissement des chaînes dans la production audiovisuelle patrimoniale.

Or la présence d'un auteur-scénariste, d'un réalisateur et de comédiens suffit au CSA pour classer Si près de chez vous dans le domaine "fiction ». Le bénéfice est double : le programme trouve son public, et la chaîne remplit son quota tout en économisant.

L'apparition de la scripted reality à la télévision française répond donc avant tout à des besoins : renouveler les émissions de journée tout en conservant un ancrage fort dans l'intime et le quotidien, et produire à bas coût. Un autre avantage a favorisé l'importation du concept allemand, celui de voir ces émissions compter dans le quota de fictions originales françaises produites par les chaînes.

L'adaptation du genre au contexte français s'est faite par contamination, hybridation avec d'autres genres télévisuels contemporains, à savoir les magazines de société et de faits divers, eux-mêmes fortement influencés par la téléréalité. Pour reprendre le schéma des différents modes énonciatifs proposés par Fr. Jost, l'écriture d'un épisode de Si près de chez s'inscrit dans une énonciation informative. Cependant la production à la chaîne d'épisodes reproduisant une même formule entre en contradiction avec ces codes et fait pencher le programme du côté de la fiction sérielle. L'effet de réel produit par des formes identifiées par le spectateur, selon sa "media literacy", comme informatives, est donc contrecarré par la standardisation des histoires: chaque fait divers réel est réécrit selon un script très précis, réagençant chaque cas et l'inscrivant dans une série qui n'a plus rien à voir avec l'inattendu et l'inhabituel du fait divers vécu. 
50 Cette mise en récit n'est cependant pas très différente des reconfigurations que les différents médias apportent à leurs réécritures des faits divers. Un article des Inrockuptibles compare ainsi la lecture du Nouveau Détective au visionnage d'un épisode de Si près de chez vous :

C'est généralement moche, mal joué, mal filmé, mais l'ensemble vite ficelé happe le téléspectateur comme il se fait prendre par la lecture rapide d'un numéro du Nouveau Détective, amusé et scotché à la fois ${ }^{51}$.

51 Au-delà de l'appréciation qualitative, cette citation évoque très justement «l'effet de réel ", de vraisemblance, fabriqué pour les lecteurs et spectateurs contemporains. Alors qu'en 1930, comme l'écrit Matthieu Letourneux, ce sont les films de gangsters qui sont convoqués dans Détective « en tant que genre de fictions produisant un effet de réel, comme si les conventions de la fiction rendaient davantage cet effet de réel ${ }^{52}$ ", créant ainsi une cohérence interne et permettant une mise en spectacle de l'événement, les « référents architextuels » contemporains sont davantage à chercher du côté de la téléréalité. François Jost souligne un paradoxe qui confirme cette impression : "on considère de plus en plus l'information comme une fiction alors qu'on croit la vérité des "show de l'authenticité", ces émissions qui font appel à la vie privée des gens ${ }^{53}$. " C'est donc sur cet intertexte que les producteurs de Si près de chez vous fondent leur effet de réel, s'inscrivant bien au cœur de la littérature médiatique qui reconfigure sans cesse selon des modes de récit différents le rapport réel/fiction.

\section{NOTES}

1. D. Maingueneau, Analyser les textes de communication, Paris, Armand Colin, 2014 : aux types de discours correspond la scène englobante ; les genres eux correspondent à la scène générique.

2. A.-Cl. Ambroise-Rendu, Petits récits des désordres ordinaires : les faits divers dans la presse française des débuts de la III République à la Grande Guerre, Paris, S. Arslan, " Histoire, cultures et sociétés ", 2004.

3. Voir Cl. Sécail, Le Crime à l'écran : le fait divers criminel à la télévision française, 1950-2010, Paris, Nouveau Monde, 2010.

B. Mariau, Écrire le fait divers à la télévision, la rhétorique émotionnelle du drame personnel au journal télévisé de TF1, thèse de doctorat en sciences de l'information et de la communication, dir. E. Souchier, Paris-Sorbonne, 2014.

4. M.-È. Thérenty, La Littérature au quotidien, Paris, Seuil, « Poétique », 2007.

5. D. Kalifa, L’Encre et le sang: récits de crimes dans la France de la «Belle Epoque» (1894-1914), Paris, Fayard, 1995.

M. Simard-Houde, Le Reporter, médiateur, écrivain et héros : un répertoire culturel (1870-1939), thèse de doctorat en littératures française et comparée, dir. M.-È. Thérenty, G. Pinson, université de Montpellier, 2015.

6. A. Vaillant, «De la littérature médiatique », dans Interférences littéraires/Literaire interferenties, nouvelle série, $\mathrm{n}^{\circ} 6$, «Postures journalistiques et littéraires », L. van Nuijs (dir.), mai 2011, p. 21-33. Voir ce passage : «je propose d'appeler littérature médiatique ce mécanisme médiatique de circulation textuelle : pour désigner non pas les catégories journalistiques qui seraient dignes 
d'une hypothétique littérarité (selon quels critères formels ?), mais, de façon bien plus générale, la communication médiatique elle-même, en tant qu'elle est, d'un point de vue fonctionnel, une forme spécifique et historiquement déterminée de la communication littéraire, ayant pris la suite de la circulation de manuscrits ou de livres imprimés, dans la société élitaire du XVIII ${ }^{\mathrm{e}}$ siècle, pour satisfaire les besoins de lecture d'un public aristocratique ou bourgeois de plus en plus nombreux » (p. 25).

7. Ibid.

8. R. Barthes, «Structure du fait divers », Essais critiques, Paris, Seuil, 1964.

9. « Du documentaire à la fiction : Quelles écritures pour parler du réel ? ", Quatrième Rencontre CNC-SACD-SCAM (Deuxième Cycle), animateur L. Heynemann, 2 juin 2008.

10. R. Wangermée, À l'école de la télé-réalité, Bruxelles, éditions Labor, 2004.

11. A. Klein et A. Tixhon, «Introduction », La Communication audio-visuelle : entre réalité et fiction, Namur, Presses universitaires de Namur, 2009.

12. La liste exhaustive donnée ici est tirée de l'article Wikipédia consacré à la scripted reality. <https://fr.wikipedia.org/wiki/Réalité_scénarisée>

13. G. Perec, L'Infra-ordinaire, Paris, Seuil, 1989 ; Fr. Jost, Le Culte du banal, de Duchamp à la téléréalité, Paris, CNRS éditions, 2007.

14. L. Henric, Les Nouvelles Stratégies de promotion, de distribution et de diffusion d'un documentaire français. Le cas particulier du docufiction à la télévision (2000-2014), thèse de doctorat en sciences de l'information et de la communication, dir. François Balle, université Paris Panthéon-Assas, 2015, p. 16.

15. A. Klein et A. Tixhon, ouvr. cité, p. 9.

16. Un programme de flux (la variété, le magazine, le divertissement, le jeu, la télé réalité, l'émission de services) est une " œuvre audiovisuelle française non patrimoniale ", c'est-à-dire qu'elle perd a priori son intérêt après sa première diffusion et ne peut constituer du stock, contrairement aux œuvres audiovisuelles françaises.

17. Fr. Jost, La Télévision au quotidien. Entre réalité et fiction, Louvain-la-Neuve, De Boeck, 2003 et «La promesse des genres ", Réseaux, volume 15, n 81, «Le genre télévisuel», 1997, p. 11-31. François Jost s'inspire du pacte de lecture pour décrire l'accord entre émetteur et récepteur à la télévision. Ce pacte de communication, que lui appelle "promesse", permet à l'émetteur d'inscrire le programme dans un genre qui est reconnu par le téléspectateur, qui peut alors enclencher la lecture adéquate. On reconnaît là l'importance du genre sur l'horizon d'attente théorisée par J.-M. Schaeffer. Cette promesse se noue dès les premières secondes du programme.

18. R. Belvaux, A. Bonzel, B. Poelvoorde, C'est arrivé près de chez vous, Belgique, Les artistes anonymes, 1992. Genre : comédie dramatique, thriller, faux documentaire.

19. On retrouve l'intégralité des deux saisons sur ces sites : <http://www.telestar.fr/programmetv/fiche-emission/si-pres-de-chez-vous-119850917/emissions> ; <http://www.programme.tv/ c9105695-si-pres-de-chez-vous/episodes/>

20. Respectivement « Privé de liberté », « Mystérieux voisins » et « L'Homme derrière les mots », Si près de chez vous, 2012.

21. Respectivement «Les Nouveaux Voisins » et « Mort au théâtre ", Si près de chez vous, 2012.

22. "Privé de liberté ", Si près de chez vous, 2012.

23. «L'Homme derrière les mots", Si près de chez vous, 2012.

24. I. Veyrat-Masson, Télévision et histoire, la confusion des genres : Docudramas, docufictions et fictions du réel, Louvain-la-Neuve, De Boeck, 2008.

25. Il faut néanmoins dès à présent nuancer cette remarque, faite à l'échelle d'un épisode : envisagé en série, puisqu'il s'agit d'une production sérielle, le script répété d'un épisode à l'autre fait pencher la balance du côté de la fiction épisodique, précisément du formula show; cette lecture s'impose d'ailleurs puisque plusieurs épisodes sont diffusés à la suite ; nous revenons sur ce point à la fin de notre développement. 
26. Le Grand Direct des Médias, Europe 1, émission du 24 novembre 2013, « La scripted-reality: "doublement intéressant" ".

M. Gasnier, «Pourquoi la scripted reality envahit nos écrans ", 15 janvier 2014, <http:// www.canal-iscpa.com/POURQUOI-LA-SCRIPTED-REALITY>

I. Mermin, «Télé : la production allemande s'impose », 29 juin 2010, <http://tvmag.lefigaro.fr/ programme-tv/article/serie/53699/tele-la-production-allemande-s-impose.html ?meId =3>

A. Gnaba " "Le jour où tout a basculé" \& Co: la "scripted reality", un spectacle du réel ", 5 novembre 2012, <http://leplus.nouvelobs.com/contribution/685299-le-jour-ou-tout-a-basculela-scripted-reality-un-spectacle-du-reel.html>

J. Henni, «Exclusif : le CSA estime que la scripted reality est de la fiction », 26 août 2013, <http:// bfmbusiness.bfmtv.com/entreprise/exclusif-csa-estime-scripted-reality-est-fiction-588726.html> Fr. Jost, "Scripted reality" et télé publique: pourquoi Filipetti a raison de s'y attaquer", 30 octobre 2012, <http://leplus.nouvelobs.com/contribution/676061-scripted-reality-et-telepublique-filippetti-a-raison-de-s-y-attaquer.html>

H. Reitzaum, E. Renault, "Courbet: "La bulle de la scripted reality va exploser" ", 28 septembre 2012, <http://www.imca.fr/actualites/julien-courbet-\%C2\%AB-la-bulle-de-la-scripted-reality-vaexploser-\%C2 \%BB/>

AFP, « Nonce Paolini (TF1) : la "scripted reality" est bien de la fiction », 6 novembre 2012, <http:// lexpansion.lexpress.fr/actualites/1/actualite-economique/nonce-paolini-tf1-la-scripted-realityest-bien-de-la-fiction_1183771.html>

27. CSA, Concertation sur les programmes dits de "réalité scénarisée », janvier 2013.

28. "Écrire des projets audiovisuels à partir de faits réels ", Troisième Rencontre CNC-SACD, modérateurs Chr. Ledannois et M. Geiger, 27 mars 2012.

29. Respectivement "Mystérieux voisins ", "L'Homme derrière les mots ", " Mort au théâtre ", «Privé de liberté », Si près de chez vous, 2012.

30. B. Mariau, Écrire le fait divers à la télévision, ouvr. cité, p. 21.

31. Ibid.

32. "Privé de liberté ", "Mystérieux voisins, "Une mère parfaite ", "L'Homme derrière les mots », Si près de chez vous, 2012.

33. "Privé de liberté », " Mystérieux Voisins ", Si près de chez vous, 2012.

34. P. Baudry, Cl. Sorbets et A. Vitalis, «Introduction », La Vie privée à l'heure des médias, Bordeaux, Presses universitaires de Bordeaux, « Labyrinthes », 2002.

35. « Mystérieux Voisins », Si près de chez vous, 2012.

36. Exemples de titres d'émissions de C'est mon choix: "Mon mari a trente ans de moins que moi », "J'impose le vouvoiement à mes enfants » (2000), cités dans Fr. Jost, Le Culte du banal, Paris, CNRS, 2007, p. 112.

37. « Mystérieux Voisins ", «L'Homme derrière les mots », « Privé de liberté », Si près de chez vous, 2012.

38. Gérard Genette parle alors de paralipse narrative (Figures III, Paris, Seuil, 1972, p. 92-93 et 212).

39. «Privé de liberté ", Si près de chez vous, 2012.

40. « L'Homme derrière les mots ", Si près de chez vous, 2012.

41. "Privé de liberté », Si près de chez vous, 2012.

42. Fr. Jost, « La promesse des genres », Réseaux, art. cité, p. 23.

43. H. Glevarec, «Trouble dans la fiction. Effets de réel dans les séries télévisées contemporaines et post-télévision », Questions de communication, $n^{\circ} 18,2010$, p. 214-238.

44. "Les programmes français considérés ici ont tous, comme leurs équivalents étrangers, un faible budget de production (de l'ordre de 1000 euros la minute) et sont produits en un temps très bref (de l'ordre de trois jours de tournage pour un « 26 minutes »). Ils sont conçus pour s'adapter aux faibles coûts de grille de journée des chaînes et se substituent, sur ces créneaux, à des séries européennes ou étrangères multidiffusées ou à des programmes de flux » (Rapport du CSA, 2013). 
45. É. Macé, Les Imaginaires médiatiques, une sociologie postcritique des médias, Paris, éd. Amsterdam, 2006, p. 120.

46. J.-P. Esquenazi, Les Séries télévisées : l'avenir du cinéma, Paris, Armand Colin, 2010, p. 26.

47. Épisode pilote, "Vengeance» propose donc le prototype que suivront les 234 épisodes produits après lui : <http://www.programme.tv/c9105695-si-pres-de-chez-vous/ vengeance-44049665/\#2>. Ce type de synopsis est récurrent : « Alors qu'il rentre chez lui, Charles, 46 ans, est blessé par un tir de fusil. Trois personnes avaient une raison de lui en vouloir »; « Hubert, 45 ans, médecin généraliste dans un village landais, est victime d'un infarctus après avoir reçu une lettre de l'Ordre des médecins qui le suspend de ses fonctions. Or trois personnes de sa commune avaient intérêt à le faire radier " (Téléstar).

48. «L'Homme derrière les mots ", Si près de chez vous, 2012.

49. Ce dispositif de trois suspects/pistes autour d'un crime est aussi celui des enquêtes en BD dans Télé7 jeux.

50. «Un épisode coûte au moins quatre fois moins cher à produire qu'un épisode d'une série classique comme Plus belle la vie (110 000 euros contre 30000 pour Si près de chez vous). » <http:// www.lesinrocks.com/2012/11/22/medias/scripted-reality-les-minifictions-vite-et-mal-tourneesqui-divisent-le-monde-la-tele-11321375/>

51. < http://www.lesinrocks.com/2012/11/22/medias/scripted-reality-les-minifictions-vite-etmal-tournees-qui-divisent-le-monde-la-tele>

52. M. Letourneux, communication prononcée lors du colloque Détective, fabrique de crimes ?, en septembre 2017 à l'université de Nîmes.

53. Fr. Jost, La Télévision au quotidien, ouvr. cité, p. 167.

\section{RÉSUMÉS}

Cet article étudie le genre télévisuel de la scripted reality ou fiction du réel à travers la série Si près de chez vous qui met en scène de petits faits divers criminels réels. Des histoires vraies sont mises en scène et jouées par des comédiens, ce qui n'a rien de nouveau dans la culture médiatique depuis le $\mathrm{XIX}^{\mathrm{e}}$ siècle. Ce qui brouille les pistes de façon nouvelle est que la réécriture utilise les styles de plusieurs médias consacrés au fait divers. À cause de cette hybridité, le statut du programme devient incertain. Nous observerons ainsi les emprunts de la réalité scénarisée à d'autres genres médiatiques, leurs effets et l'enjeu de tels programmes dans la grille télévisuelle.

This article is about the scripted reality, focusing on Si près de chez vous, a French TV show about real criminal facts. True crime stories are played by actors, which is quite usual since the $\mathrm{XIX}^{\text {th }}$ century and the mediatic culture. What is new is that these episodes are written with journalistic technics, making the spectator hesitate between fact and fiction. We will observe how and why these technics are used and we will question the aim of those programs in the making of the today television. 


\section{AUTEUR}

\section{AMÉLIE CHABRIER}

Université de Nîmes, CHROME (EA 7352), RIRRA 21 (EA 4209).

Amélie Chabrier est maître de conférences en littérature française à l'université de Nîmes, membre de CHROME et membre associée du RIRRA 21. Sa thèse porte sur Les Genres du prétoire, littérature et chronique judiciaire au XIX ${ }^{e}$ siècle. Ses recherches concernent la culture médiatique et les liens entre presse et littérature, depuis la presse francophone des années 1930 jusqu'à des objets plus contemporains comme les séries télévisées. Ses travaux les plus récents sont Détective, fabrique de crimes? co-écrit avec Marie-Eve Thérenty, Nantes, Joseph K., janvier 2017 ; « Veni, bibi, vici : les aventures extraordinaires de Mrs Eleanor Holm Jarret », dans « Les JO 1936 dans la Presse francophone », Belphégor, 2017 ; ou encore « Les Mystères de New York: du serial américain au roman-cinéma français : l'ombre de Sue ? ", Rocambole, nº 78-79, printemps-été 2017, p. 41-56. 